\title{
14
}

\section{Quality Management as Basis of Environ- mental Management Systems for Industrial Plants}

\author{
P.H. OSANNA *), D. PROSTREDNIK *), A. OSANNA **)
}

*) Department for Interchangeable Manufacturing and Industrial Metrology (Austauschbau und Messtechnik), Institute of Production Engineering, Vienna University of Technology - TUW, Karlsplatz 13/3113, A-1040 Wien, Austria.

**) Department of Physics, State University of New York at Stony Brook, Stony Brook, NY 11790-3800, USA.

\begin{abstract}
Quality management systems according to International Standards of the ISO 9000 series are a good basis for building environmental management practice. This offers the opportunity to apply well known systems and well known tools in a modified form to solve a company's environmental problems. Additionally, this will put the environmental question far beyond national boarders maybe throughout the world.

Legislation, consumers and environmental organisations put increasingly pressure on companies to improve their environmental performance. Therefore in various countries adequate managersent of environmental issues is becoming indespensible as a means to keep a companies competitive position.
\end{abstract}

Keywords

environmental management systems, quality management systems, small and medium sized enterprises

\section{$1 \quad$ WHAT IS OUR POINT OF VIEW ?}

Quality management based on ISO 9000 is a very advanced and efficient management system in various countries all over the world. The know-how and experience in quality management derives of the early years of twentieth century and since that time developed continously. Today a well developed quality management system is a key factor of every company's 
success. We find quality management systems in all kinds of industrial plants and economical structures and also other organisations according to (Osanna, 1991).

Legislation, consumers and environmental organisations are increasingly putting pressure on companies to improve their environmental performance. Thus, in an also increasing number of countries adequate management of environmental issues is becoming indispensable as a means to keep a company's competitive position.

\section{CAN WE INTERCORRELATE ENVIRONMENTAL AND QUALITY ISSUES ?}

Besides controlling the quality of products the management system on the basis of the series of International Standards EN ISO 9000 especially (EN ISO 9000-1, 1994) and (EN ISO 9004-1, 1994) can build in a similar way the basic system for handling the interactions between a company and the needs of ecology. The starting point for a comparison of systems must be to find the joint characteristics of quality and environmental issues.

An obvious possibility to combine quality and environmental issues is given by the definition of quality. According to the term standard (EN ISO 8402, 1994) quality is defined as the entity of features of a unit referring to the appropriateness to fulfill determined and premised requirements. Nowadays in industrialized countries it is widely agreed that the environmental compatibility of a product is a quality feature. But it is not a customer-specific, it is a more or less society-specific quality feature. The only question arising in this context is: How much are customers willing to pay for this "society-specific quality feature" environmental compatibility?

There is another reason for the link between environmental and quality management. The quality management system according to the EN ISO 9000 series was developed to satisfy costumer demands for quality. But the management principles are generally valid and can be used to solve and manage other society interests. In the same way, how technical specifications for quality are separated from the management system for quality, the technical specifications in these areas have to be developed separately too.

This separation of management system standard and different environmental standards which depend on a lot of heterogeneous regulating actions in various countries and industrial branches - is an essential boundary condition.

These general ideas have been taken as a basis for the development of an environmental management system by the British Standards Institute as the standard (BS 7750, 1992) on the roots of the EN ISO 9000 series.

\section{WHAT IS THE EUROPEAN POINT OF VIEW ?}

At present there exist two different system approaches in Europe.

On the one hand the EC in general focusses systems on auditing of internal management systems as well as the publication of the results and a verification on this bases.

On the other hand according to (BS 7750, 1992) an all including management system should be developed and implemented which later on should be ready for audits. At present this approach is often entitled as the "ultimate standard". In reality it is more than just the sum of 
the two parts "quality" and "environmental management", it is a compound of both perspectives by taking the largest synergetic effects possible.

On this basis new proposals for international standards give general ideas for the principles of environmental management systems according to (ISO 14000, 1994) and guidance for their use according to (ISO 14001, 1994).

\section{WHAT CAN WE LEARN FROM THE QUALITY CIRCLE ?}

Environmental problems can be handled only in a co-ordinated and systematic way by developing straight mechanisms. This has been realised lately by proactive companies.

The function overlapping integration gives a chance to recognise problems very early. In this way a minimisation of risks and a maximisation of chances is given.

Although the critical phases of a products live are very different within various industrial sectors, in a global overlook according to (McKinsey \& Co., 1991) production and disposal/recycling have the strongest impact on the environment as Table 1 shows.

Table 1 Critical phases of product life by sector, in $\%$

\begin{tabular}{lcccc}
\hline & Sourcing & Production & Product use & Disposal/recycling \\
\hline Average: & 11 & 47 & 13 & 29 \\
\hline Chemicals & 10 & 49 & 21 & 20 \\
Energy & 13 & 58 & 16 & 13 \\
Metals & 8 & 62 & 3 & 27 \\
Process industry & 11 & 41 & 11 & 37 \\
Durables & 16 & 24 & 25 & 35 \\
Consumer goods & 7 & 45 & 9 & 39 \\
\hline
\end{tabular}

The idea of a value chain is a systematical identification of ecological problems through all functions or parts of a company. The three basic functions - sourcing, production and distribution - include all company's functions. These three functions - added by all interfaces can be very useful to build the basis for material balances as well as process and product balances for the problem identification. The value chain analysis is a diagnosis tool with its aim on cost reduction an yield increase.

The quality circle according to (EN ISO 9004-1, 1994) is a phase concept developed on all processes within a company (see Figure 1). As not only costs are in the point of view the interactions between the different stations are taken into consideration too. Therefore the result is a closed phase loop as Figure 1 shows.

Given by the models background of the involvement of all phases of a products life with its interfaces to the environment, the model is not only very universal it is also very useful for the analysis and optimisation of methods and processes in a single function or part of a company. 


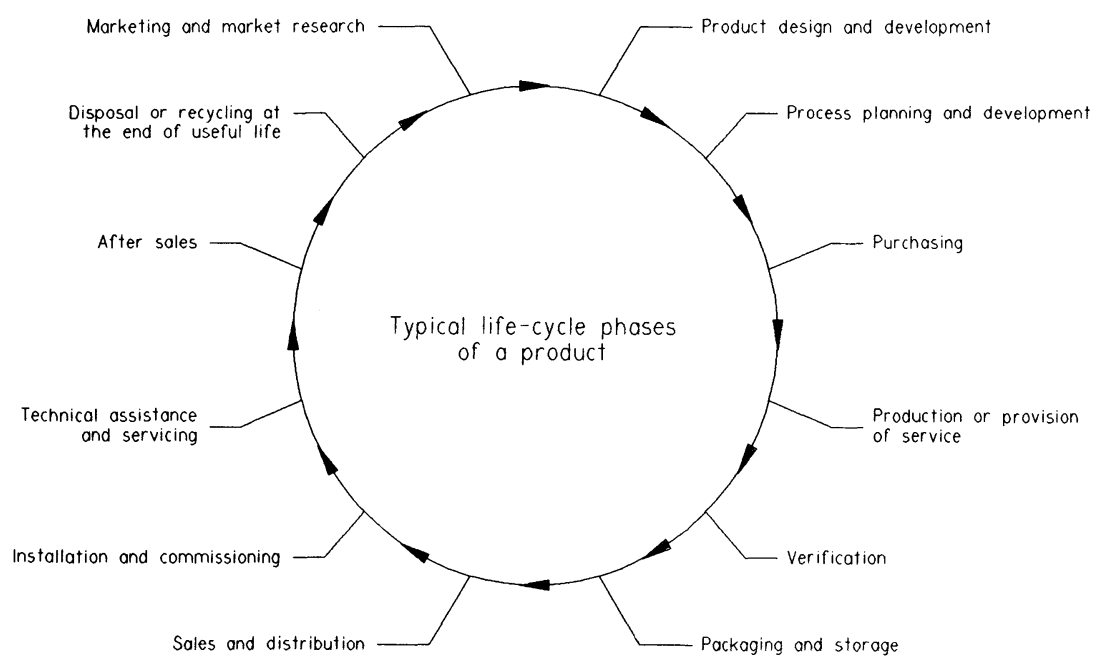

Figure 1 Quality circle.

\section{WHAT SHOWS THE COMPARISON OF MANAGEMENT SYSTEMS ?}

One of the most important actions on the way to an integrated environmental management system is the organizational embedding of environmental thoughts in a company's organisation based on an environmental strategy.

\subsection{Philosphy, strategy and policy}

The philosophy of a company includes the common aims and values within this organisation. Thus it is the starting point for the determination of the whole system.

Environmental ideas of companies are mostly formulated with reference to specific addresses for the society. With this the growing environmental awareness of the society was taken into account in the past. But facing growing environmental problems in the 1980's few companies widened their principles. Nowadays an exellent example for such principles is formulated in the ten guidelines of an already published environmental codex (International Chamber of Commerce, 1991). These can be easily adopted to a company-wide guideline for environmental care.

A strong similarity of these guidelines to the statements of quality management is evident. Both request an involvement of all relevant actions and tools within the policy, the possibility of public information and a system enclosing all hierarchical levels with the goal to provide continuous improvement. These guidelines leading to an overall principle for the company aim on an innovative environmental strategy. 
Although there are a lot of different strategies discussed within publications they all can be reduced to three strategies. Figure 2 shows the matrix of these strategies which have different meanings within different industries and branches but they imply the same system.

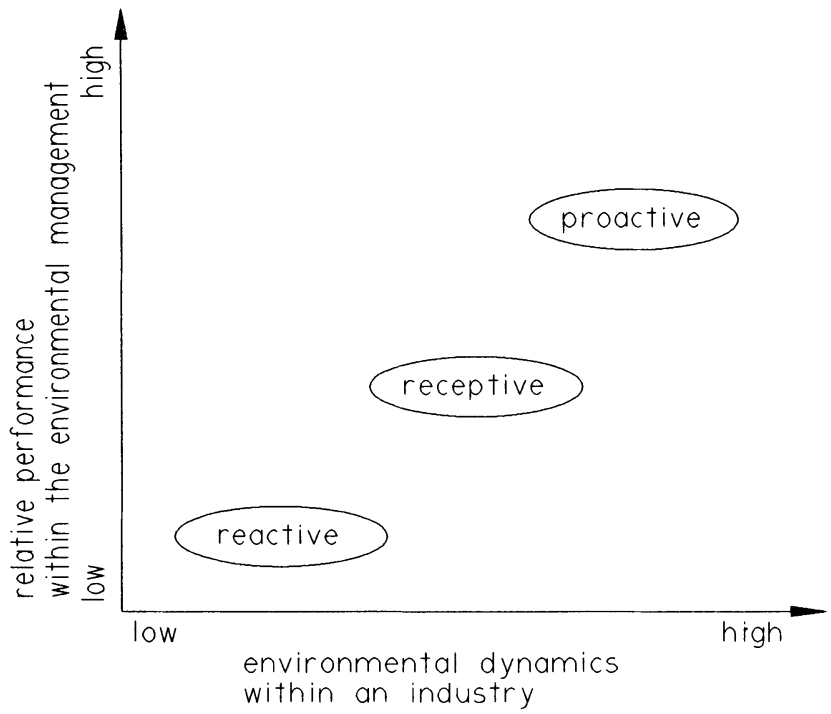

Figure 2 Matrix of environmental strategies.

\subsection{Organizational structure}

On the one hand as environmental care needs the participation of all employees the sequel is that there is no need for institutionalisation of environmental tasks because the implementation of such an environmental department takes away the personal responsibility for environmental tasks from the employees.

On the other hand the complexity of environmental decisions is a good reason for the centralisation of environmental planning and decision making on a higher hierarchical level. But this can lead to an overtax of this organisational level.

For the institutionalisation of environmental tasks can be given several reasons according to (Heiss, 1994):

- reduction of complexity

- balancing function

- control function

- innovation function.

A useful proposal for the embedding environmental functions shows Figure 3. 


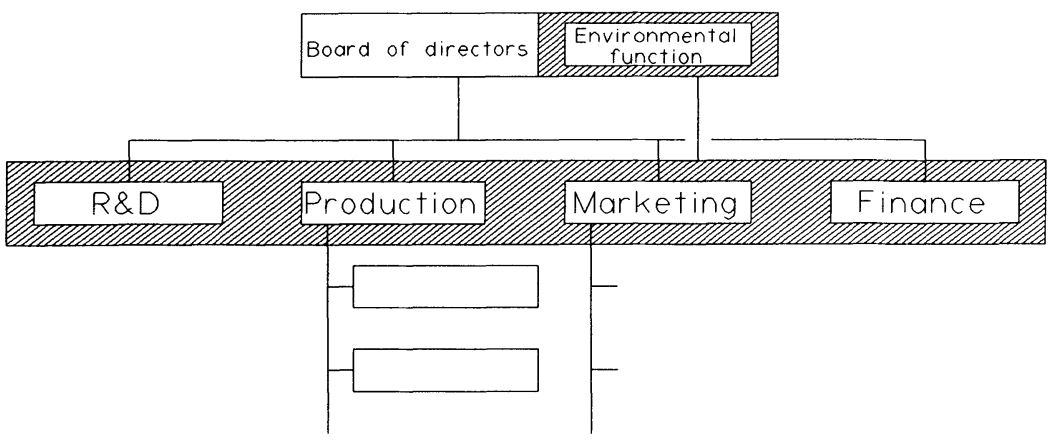

Figure 3 Proposal for the organisational company structure.

\subsection{Quality circle and environmental circle}

The idea behind "quality circles" in this context is that problems are most easily recognised and eliminated at their point of origin. This means that the possibility to solve problems within their working place is given to employees and supported by the membership of a problem-solvinggroup. As the name "quality circle" taken from the Japanese model of "quality control circles" is no longer state of the art - because nowadays this concept is in most cases no more used for quality relevant issues - we speak in this context of problem-solving-groups.

Environmental care within a company is only efficient if it is supported by everyone and all the small actions, which as a sum are a key-success-factor of an environmental quality management system, are done by each employee. Problem-solving-groups with specific environmental tasks are called "environmental circles".

- The implementation of environmental circles can bring a series of advantages:

Everyone is confronted and involved with environmental problems and therefore as part of the new philosophy and system involved persons are motivated.

- Enormous human capital is set free for environmental topics.

- A lot of small weak points are in sum a huge potential of failure and costs.

\section{$5.4 \quad$ Audits}

In the above mentioned publication (International Chamber of Commerce, 1991) a good definition of environmental auditing is given as a "management tool comprising a systematic, documented, periodic and objective evaluation of how well environmental organisation, management and equipment are performing with the aim of helping safeguard the environment".

This can be achieved by:

- facilitating management control of environmental practices and

- assessing compliance with the company's policies which include meeting regulatory requirements. 
There are similar definitions given in the new proposals for international standards (ISO 14000,1994 ) and (ISO 14001, 1994). A strong correspondence to the definition for quality audits according to (EN ISO 9004-1, 1994) is obvious.

Additionally it must be taken into consideration that there exist also proposals for legal regulations for the auditing of environmental systems e.g. (Austrian Draft Law, 1994).

\section{CAN WE DRAW ANY CONCLUSIONS ?}

The advantages of an environmental management system based on a quality management system is not only the application of a widely accepted and approved system. Additionally, various efficient tools and methodes are ready for use.

On the basis of the guidelines for international quality management system a frame system for environmental issues can be easily established and implemented in a company. Many elements can be derived directly from quality management, other already established modules can be installed easily.

The joint characteristics of quality and environmental issues are the point of view of this paper. Different basic concepts and methods for subsolutions within the system are discussed and possibilities for implementation are proposed. Further the environmental management system with its system parts and the common use of some important quality tools in a modified form can build the basis for a system to handle the interactions between a company and its environment.

In this way it is possible to find an effective but low cost solution for an environmental management system especially under the point of view of small and medium sized enterprises. Recently published draft standards e.g. (BSI, 1994) show the possibility that the general ideas given in this paper may be of help for future tasks of organisations and enterprises in the field of health and security.

\section{REFERENCES}

Austrian Draft Law (1994) Bundesgesetz ueber die Zulassung von und die Aufsicht ueber Umweltgutachter sowie ueber die Fuehrung des Standortverzeichnisses entsprechend dem EU-Gemeinschaftssystem fuer das Umweltmanagement und die Umweltbetriebspruefung - Oeko-Audit-Gesetz. 144761/7-II/C/5/94.

BS 7750 (1992) Specification for Environmental Management Systems.

BSI (1994) Guide to Health and Safety Management Systems. Draft Standard, 94/408875DC.

EN ISO 8402 (1994) Quality Management and Quality Assurance - Vocabulary.

EN ISO 9000-1 (1994) Quality Management and Quality Assurance Standards - Part 1:

Guidelines for Selection and Use.

EN ISO 9004-1 (1994) Quality Management and Quality Systems Elements; Guidelines.

Heiss, C.P. (1994) Managementloesungen fuer die Umwelt durch angepasstes Qualitaetsmanagement. e \& i, 111, 4, 189-192.

International Chamber of Commerce (1991) ICC Guide to Effective Environmental Auditing. ISO 14000 (1994) Guide to Environmental Management Principles, Systems and Supporting Techniques. ISO/TC 207/SC 1 N 48, Committee Draft. 
ISO 14001 (1994) Environmental Management Systems - Specification with Guidance for Use. ISO/TC 207/SC 1 N 47, Committee Draft.

McKinsey \& Co. (1991) The Corporate Response to the Environmental Challenge. Summary Report.

Osanna, P.H. (1991) Qualitaetssicherung und Qualitaetsmanagement an der Universitaet. QZ Qualitaet und Zuverlaessigkeit, 36, 8, 459-464.

\section{BIOGRAPHY}

Prof. Dr. P.Herbert OSANNA is head of the Department for Interchangeable Manufacturing and Industrial Metrology (Austauschbau und Messtechnik) at the Institute of Production Engineerin, Vienna University of Technology (TUW, Karlspl.13/3113, A-1040 Wien, Austria). $\mathrm{He}$ is author of more than 150 scientific publications and he has given more than 150 lectures in Europe as well as in USA and Asia.

Dr. Daniel PROSTREDNIK studied at the Technical University of Bratislava. Since 1993 he is member of the staff of the Department for Interchangeable Manufacturing and Industrial Metrology.

Magister Angelika OSANNA, M.A., graduated in Physics at the University of Vienna in 1992. Since 1993 she is at the Department of Physics at the State University of New York at Stony Brook (SUNY, Stony Brook, NY 11790-3800, USA). 